\title{
The Significance of Gingivitis During the Maintenance Phase of Periodontal Treatment*
}

\author{
E. C. Morrison, † S. P. Ramfjord, F. G. Burgett, R. R. Nissle, and \\ R. A. Shick
}

Accepted for publication 9 February 1981

\begin{abstract}
THE SIGNIFICANCE of recurrent gingivitis related to maintenance of post-treatment pocket depth and clinical attachment levels was tested in 78 patients over 7 years of maintenance care with prophylaxis every 3 months. Under the experimental conditions the severity of the recurrent gingivitis did not significantly affect the pocket depth and attachment levels.
\end{abstract}

It has become increasingly evident during the last decade that the intracrevicular bacterial flora is quite different in gingivitis and periodontitis. ${ }^{1,2}$ The crevicular bacterial flora also is radically changed by treatment of periodontal pockets, ${ }^{3,4}$ and several months may be required for the bacterial flora in a periodontal pocket to return to the pretreatment state. ${ }^{3-5}$ A slow subgingival penetration of supragingival plaque also has been reported in teeth extracted at various time intervals after scaling. ${ }^{6}$ It thus may be that supragingival plaque may form and develop marginal gingivitis for some time without concomitant reaction of periodontitis at the bottom of a treated periodontal pocket. It also has been reported that clinically measurable pocket depths and attachment levels may be maintained essentially unaltered in treated pockets with less than perfect plaque control $^{7}$ and some gingivitis as long as the patient is on a 3-month recall and prophylaxis program. ${ }^{8}$

The obvious question is whether the severity of the recurrent gingivitis has any influence on pocket depth and attachment level in treated periodontal patients on recall for prophylaxis every 3 months. A clinician tends to be more concerned with a previously treated area with severe gingivitis at the time of the recall than with an area of mild gingivitis. However, the fact that we found no variations in pocket depth and attachment levels related to plaque scores ${ }^{7}$ in such recall patients may suggest that the severity of gingivitis also has no influence on clinical pocket depth and attachment levels.

\section{MATERIALS AND METHODS}

The present paper is based on the same material as

* This study was partially supported by United States Public Health Service Grant DE 02731.

$\dagger$ Department of Periodontics, The University of Michigan, School of Dentistry, Ann Arbor, MI 48109. the previous paper on plaque control. ${ }^{7}$ The methods are also the same, except that this paper is concerned with gingivitis which was scored according to the PDI method. ${ }^{9}$ The gingivitis scores 1 year post-treatment were used for comparison of pocket depth and attachment responses to various categories of gingivitis during 7 years of maintenance therapy.

First, the material was divided into median and lower gingivitis scores, as against higher than median scores. Individuals with the upper $25 \%$ of gingivitis scores also were compared with those having the lower $25 \%$ of scores, to permit a clearer separation of responses if the gingivitis scores should have an impact. Students $t$ test was used to compare the pocket and attachment responses in the two categories of gingivitis scores.

\section{RESULTS}

When pocket reduction for pockets 1 to $3 \mathrm{~mm}$ was related to maintenance gingivitis scores (Fig. 1), there was no difference 1 year after treatment, and the gradually developing difference over time indicated more return of pocket depth for the lower than for the higher gingivitis group. However, when the $25 \%$ with the lowest

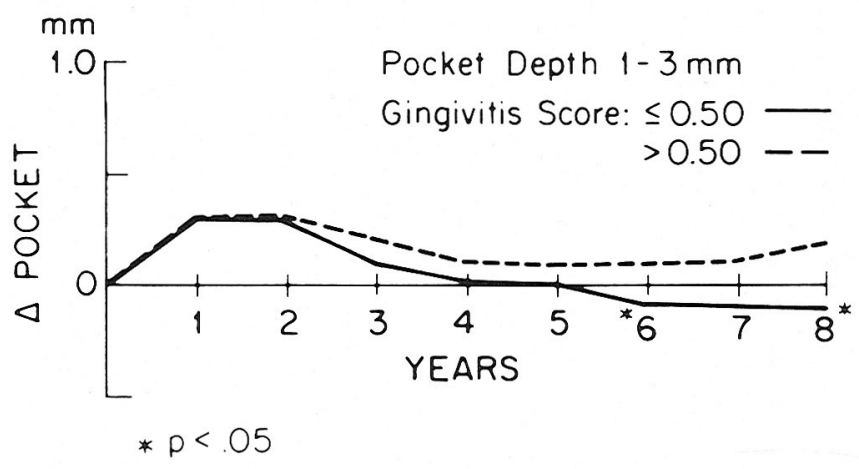

Figure 1. Pocket reduction related to Gingivitis over 8 years. 
gingivitis scores were compared with the $25 \%$ with the highest gingivitis scores (Fig. 2), there was no difference, thus negating the findings in Figure 1. With regard to attachment levels for the 1 to $3 \mathrm{~mm}$ pockets (Figs. 3 and 4) there appeared to be slightly less loss of attachment 2 years post-treatment for the groups with the lower gingivitis scores, but from then on there was no difference until the end of the study.

Considering the initial pockets of 4 to $6 \mathrm{~mm}$ (Figs. 5 and 6), there was no difference in pocket response for the first 5 years post-treatment, and after that the pocket reduction was better maintained in the groups with the higher gingivitis scores. However only a couple of these differences were significant statistically and even they were not significant when the upper and lower $25 \%$ are compared (Fig. 6).

With regard to clinical attachment changes in the 4 to

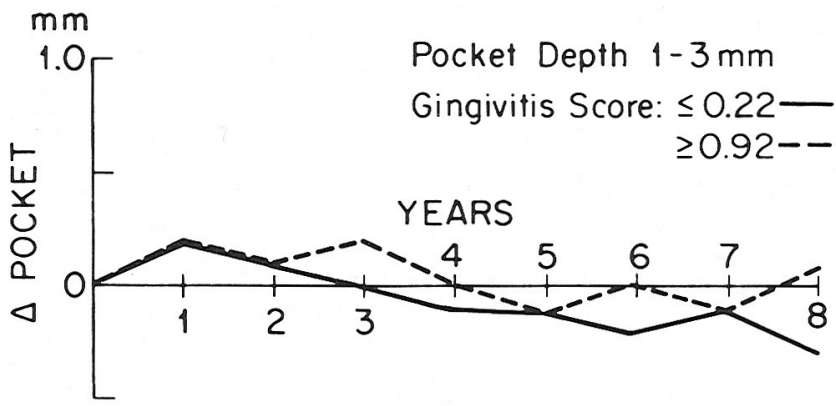

Figure 2. Pocket reduction related to Gingivitis over 8 years.

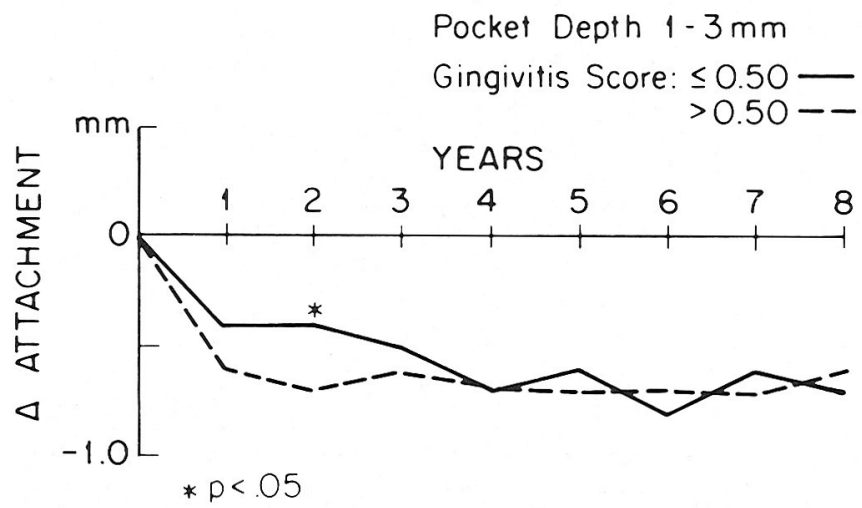

Figure 3. Attachment change related to gingivitis over 8 years.

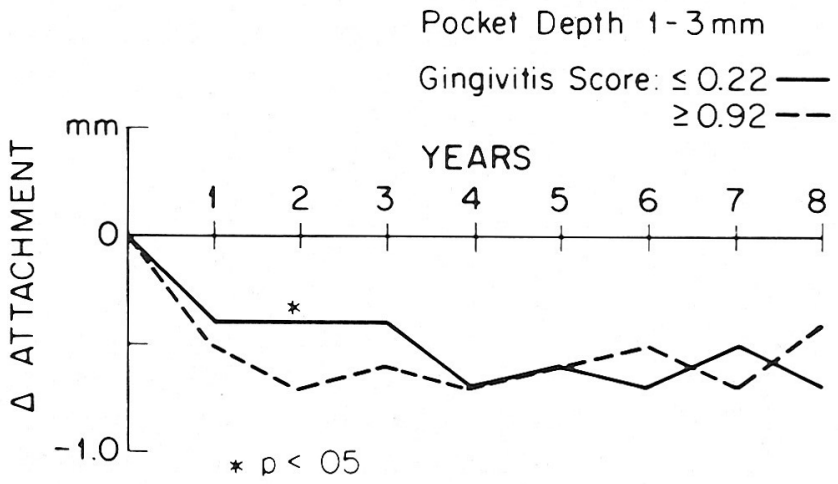

Figure 4. Attachment change related to gingivitis over 8 years.

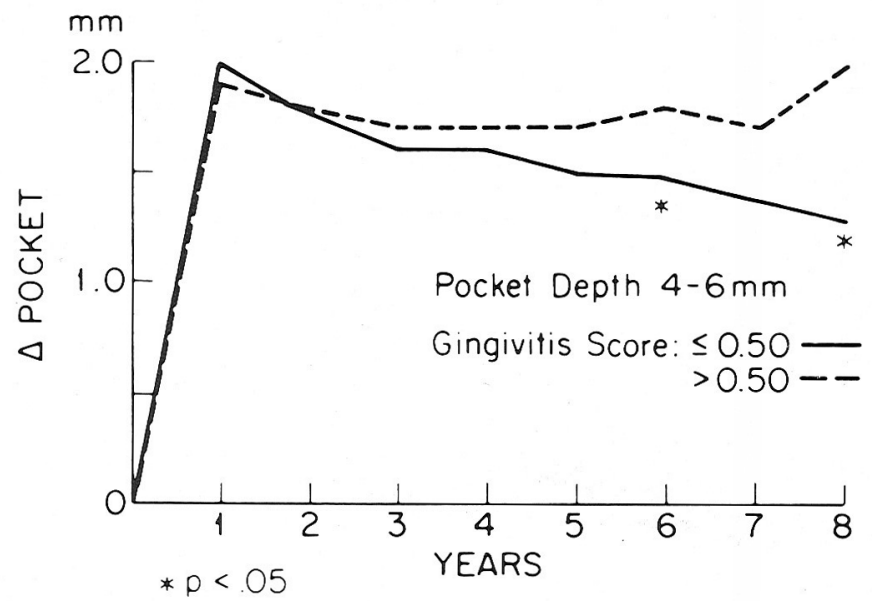

Figure 5. Pocket reduction related to gingivitis over 8 years.

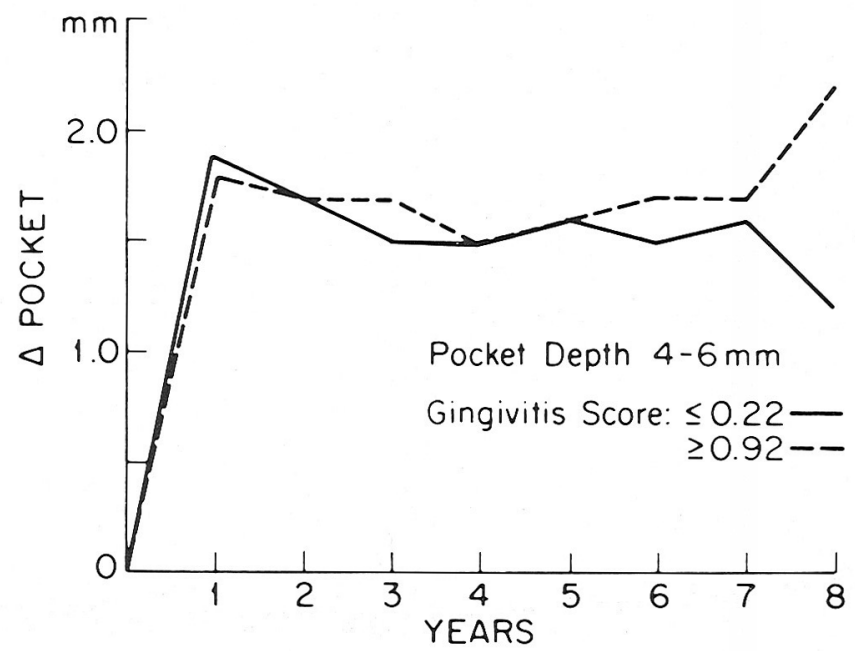

Figure 6. Pocket reduction related to gingivitis over 8 years.

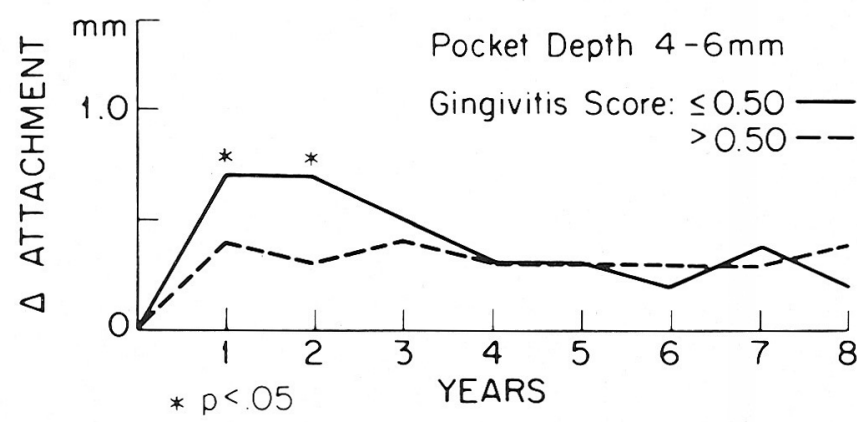

Figure 7. Attachment change related to gingivitis over 8 years.

$6 \mathrm{~mm}$ pockets (Figs. 7 and 8), there appeared to be a greater gain in attachment for the low gingivitis groups for the first 2 years post-treatment, but from that time to the end of the experiment there were no differences.

For the pockets $\geq 7 \mathrm{~mm}$ (Figs. 9 and 10), there appeared to be more pocket reduction for the groups with the least gingivitis, although the differences were significant statistically only for 3 years. The differences were initiated during the 1st year after treatment, indicating a favorable initial treatment reponse related to low gingivitis scores, rather than to an impact during the 


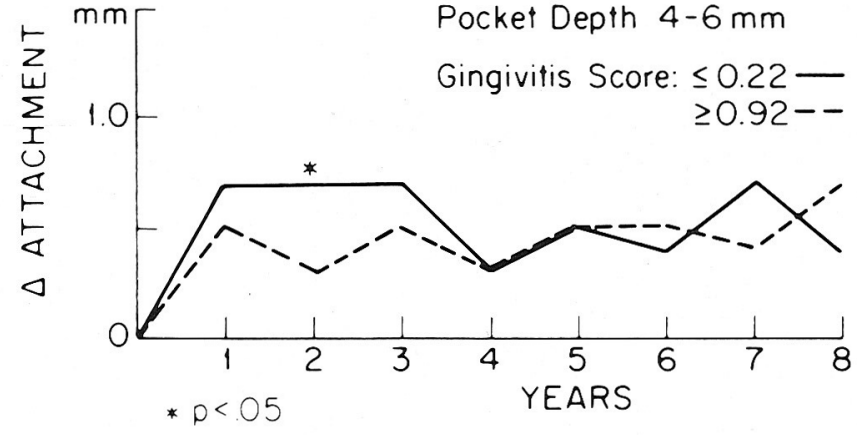

Figure 8. Attachment change related to gingivitis over 8 years.

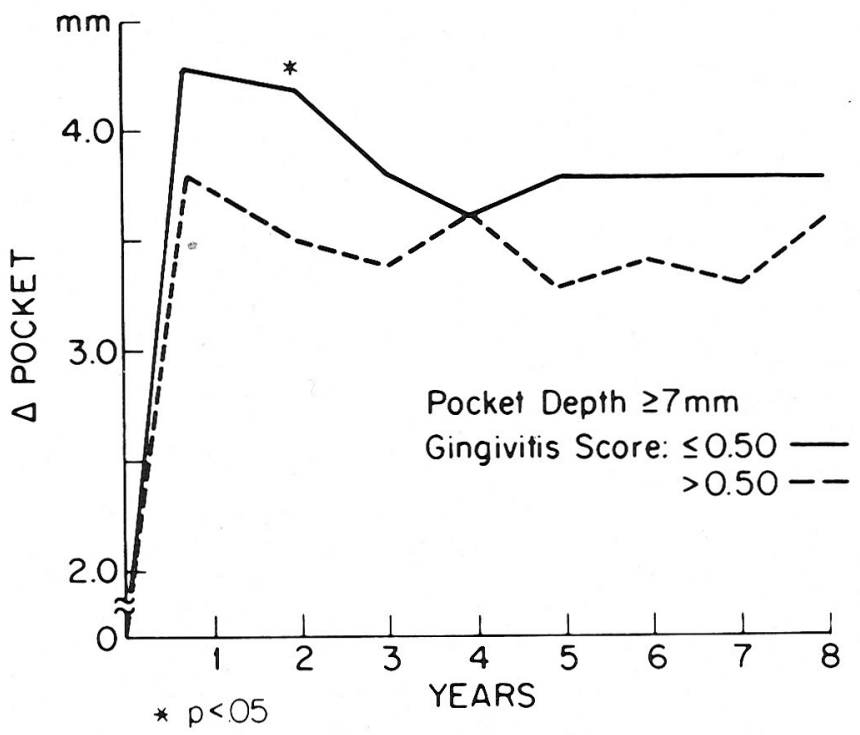

Figure 9. Pocket reduction related to gingivitis over 8 years.

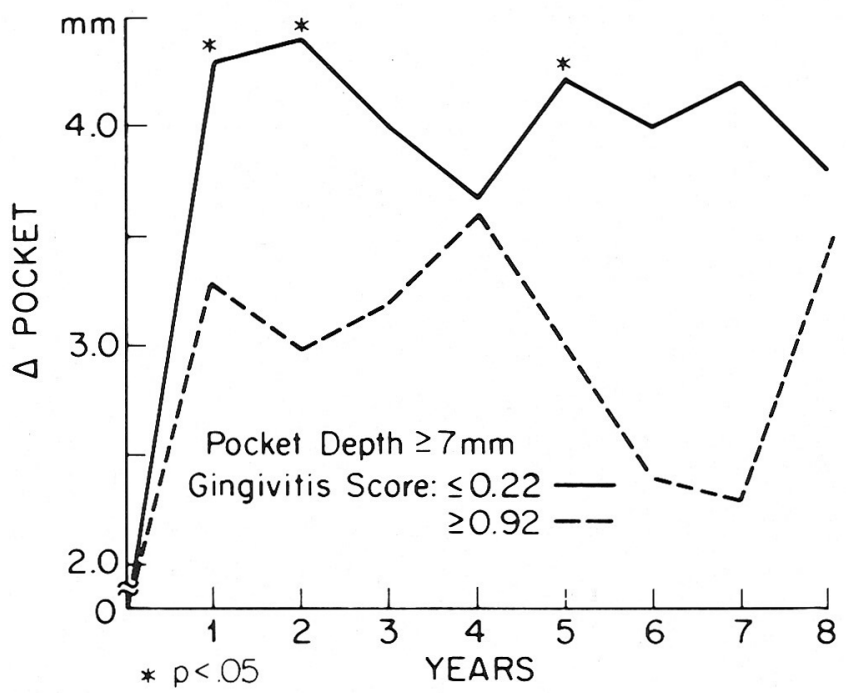

Figure 10. Pocket reduction related to gingivitis over 8 years.

maintenance years where the two curves ran a comparable course for most of the time.

When the variations in clinical attachment over time in $\geq 7 \mathrm{~mm}$ pockets (Figs. 11 and 12) were related to gingivitis scores, there appeared to be a more favorable response associated with low gingivitis scores. Marked

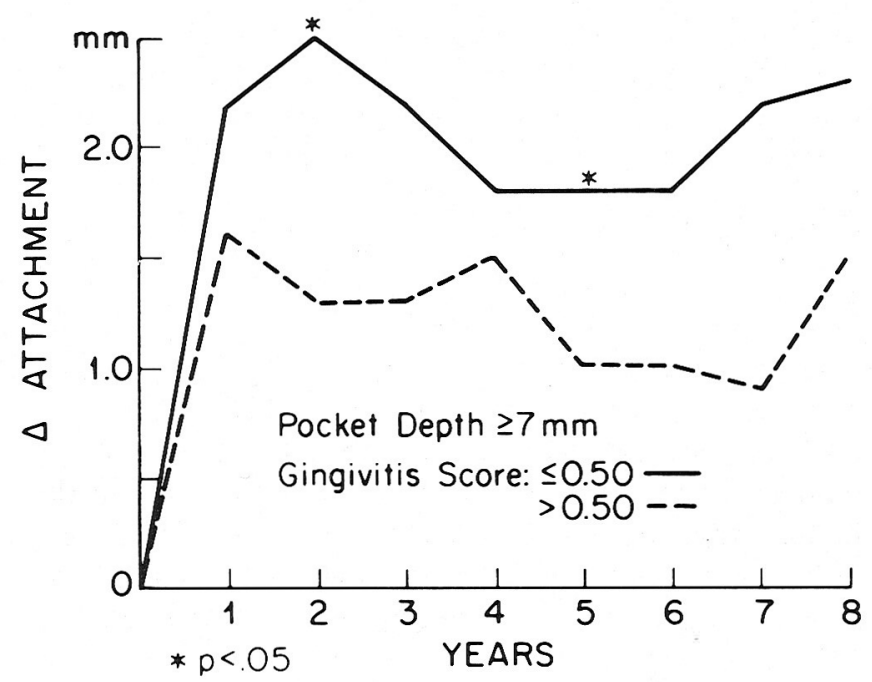

Figure 11. Attachment change related to gingivitis over 8 years.

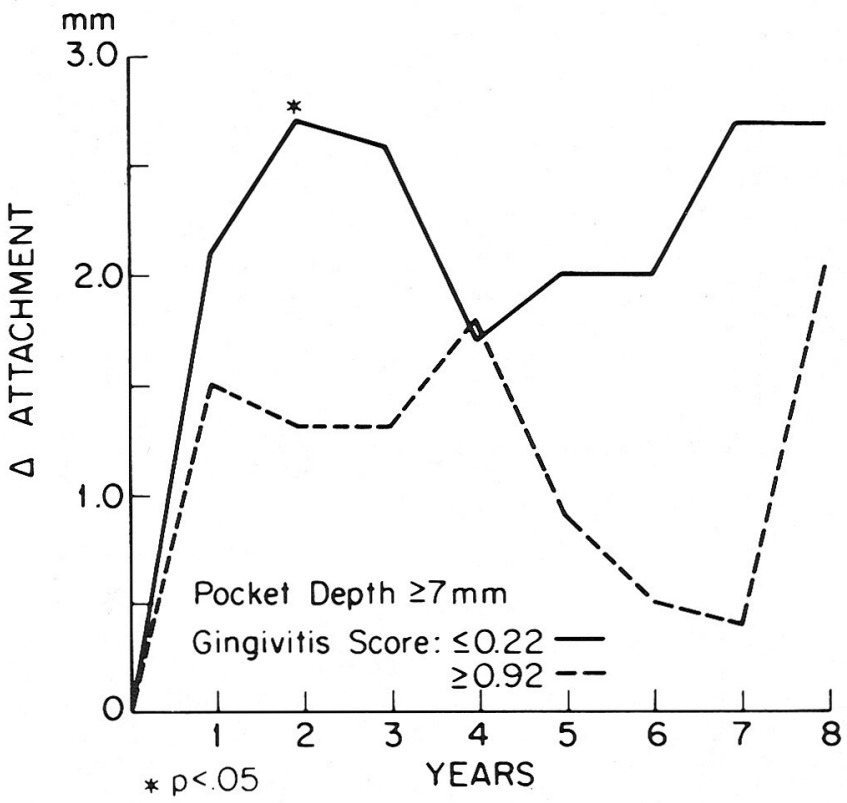

Figure 12. Attachment change related to gingivitis over 8 years.

individual variations in the few subjects with pockets $\geq$ $7 \mathrm{~mm}$ accounted for the somewhat erratic course of the curves, and as in the case of the pocket reductions in this category, the differences in attachment responses appeared basically to be related to a more favorable initial response after treatment for the groups with lower gingivitis scores.

\section{SIGNIFICANCE}

The gingivitis score in the PDI index is based on scores for separate teeth, but not for separate surfaces of the teeth, so no specific correlation study can be made based on our data between gingivitis and pocket depth or attachment level variations related to specific sites on a tooth. The basis for our comparison was the patient's mean response for pockets of a certain depth related to the patient's overall mean gingivitis score during 7 years 
of maintenance care. It also should be noted that the mean gingivitis score during the maintenance phase was low (0.5), with a rather low range of 0.1 to 2.0. Thus, there was no great difference between the lower than median and higher than median scores. Dividing the material in upper and lower $25 \%$ scores separated the groups more, but even then the scores of 0.22 and 0.92 still were far below the median of the PDI index of 1.38 (range 0.1-3.0) at baseline. This indicated that the index was not ideal for recording the small variations in gingivitis after treatment that were seen in this population. Thus, the results have to be interpreted with some reservations, and we can not exclude the possibility that real differences may have been masked.

On the basis of the available material, it appears that there was no consistent relationship between degrees of gingivitis and variation in the clinical parameters of pocket depth or attachment level during 7 years of maintenance care with prophylaxis every 3 months. However, the tendency was for pockets $\geq 7 \mathrm{~mm}$ and deeper to show more initial pocket reduction and gain of attachment in patients with lower than median gingivitis scores than in patients with higher than median scores. However, if the comparison is limited to the maintenance period between year 1 and year 8 there is not much difference in the curves, which appear to be erratic, partially due to a small number of patients with such deep pockets.

The total impression is that the severity of mild recurrent gingivitis during maintenance care with prophylaxis every 3 months has little if anything to do with maintenance of pocket depth and attachment levels. However, the result of the initial treatment in terms of reduction in pocket depth and gain of attachment in deep pockets may be enhanced in patients with low gingivitis scores.

These results are very similar to the results from our plaque study in the same material, as might be expected from the known close correlation between supragingival plaque and gingivitis scores. ${ }^{10,11}$

\section{CONCLUSIONS}

Severity of recurrent gingivitis during maintenance therapy with prophylaxis every 3 months does not appear to have any significant impact on recurrence of pocket depth or maintenance of clinical attachment levels.

\section{REFERENCES}

1. Socransky, S. S.: Microbiology of periodontal disease. Present status and future considerations. J Periodontol 48: 497, 1977.

2. Listgarten, M. A., and Hellden, L.: Relative distribution of bacteria at clinically healthy and periodontally diseased sites in humans. J Clin Periodont 5: 115, 1978.

3. Listgarten, M. A., Lindhe, J., and Hellden, L.: The effect of tetracycline and/or scaling on human periodontal disease. Clinical microbiological and histological observations. $J$ Clin Periodont 5: 2, , 1978.

4. Syed, S. A., Morrison, E. C., Loesche, W. J., and Ramfjord, S. P.: The bacterial flora of treated periodontal pockets. I.A.D.B. Abstract No. $76,1980$.

5. Loesche, W. J.: Chemotherapy of dental plaque infection. Oral Sci Rev 9: 65, 1976

6. Waerhaug, J.: Healing of the dento epithelial junction following subgingival plaque control. II. As observed in extracted teeth. $J$ Periodontol 49: 114, 1978.

7. Ramfjord, S. P., Morrison, E. C., Burgett, F. G., Nissle, R. R., Shick, R. A., Zann, G. J., and Knowles, J. W.: Oral hygiene and maintenance of periodontal support. (Submitted for publication).

8. Knowles, J. W., Burgett, F. G., Nissle, R. R., Shock, R. A., Morrison, E. C., and Ramfjord, S. P.: Results of periodontal treatment related to pocket depth and attachment level. Eight years. J Periodontol 40: $225,1979$.

9. Ramfjord, S. P.: Periodontal disease index (P.D.I.). $J$ Periodontol 38: $602,1967$.

10. Naylor, G. G.: Correlation among the Ramfjord plaque index, the Ramfjord gingivitis index, and dry weight plaque. Thesis. The University of Michigan, Ann Arbor, 1974.

11. Russell, A. L.: Epidemiology of periodontal disease. Int Dent $J$ 17: $282,1967$.

Send reprint requests to: Dr. S. P. Ramfjord, The Department of Periodontics, The University of Michigan, School of Dentistry, Ann Arbor, MI 48109. 